\title{
LINEAR RECURRING M-ARRAYS
}

\author{
Dongdai Lin, - Mulan Liu \\ Institute of Systems Science, Academia Sinica \\ Beijing, 100080, China
}

\section{ABSTRACT}

In this paper, the properties, structures and translation equivalence relations of linear recurring m-arrays are systematically studied. The number of 1 inear recurring m-arrays is given.

\section{Intrduction}

Reed and Steward [11], Spann [5] and [2] have studied the arrays of so-called perfact maps. This has led co research on various types of window properties for arrays (see [2]-[11]).

In this paper, we make a systematic study of the linear recurring m-arrays of dimension 2. We characterize their structure, discuss their properties of transiation - addition, pseudo-random and sampling. We also give the number of linear recurring m-arrays.

All the results in this paper are obtained over the finite field GF(2). One can easily generalize the results to any finite field GF(q).

\section{Basic concepts}

Let $A=\left(a_{i j}\right)_{i \geqslant 0, j \geqslant 0}$ be $a n$ array. An mxn submatrix $A(i, j)=\left(a_{i j}\right)_{0 \leqslant i<m, 0 \leqslant j<n}$ of $A$ is called an mxn window of $A$ at $(i, j) . \bar{A}(i, j)$ is the row vector ( $\left.a_{t}\right){ }_{b t<m n}$ of dimension $m n$, where $a_{t}=a_{i+i^{\prime}, j+j^{\prime}}, i^{\prime}=t$ he integer part $[t / n]$ of $t / n$, and $j^{\prime}=(t / n)=t-n[t / n]$.

Definition 2.1: Let $A$ be an array of period rxs. If all mxn windows in a period of A are exactly all non-zero mxn matrices over GF(2), then we call $A$ an mxnth order m-array of period rxs or $(r, s ; m, n)$ m-array in short.

Corollary 2.1.1: There exists an $(r, s ; m, n) m-a r r a y$ only if $r s=2^{m n}-1$.

Definition 2.2: Let $A=\left(a_{i j}\right)$ i $\geqslant 0, j \geqslant 0$ be an array, m and $n$ are two positive integers. If there exist two mnxmn matrices $T_{h}$ and $T_{v}$ as in (2.2) such that

$$
\begin{aligned}
& \bar{A}(i, j) T_{h}=\bar{A}(i, j+1) \\
& \bar{A}(i, j) T_{v}=\bar{A}(i+1, i)
\end{aligned}
$$

and 


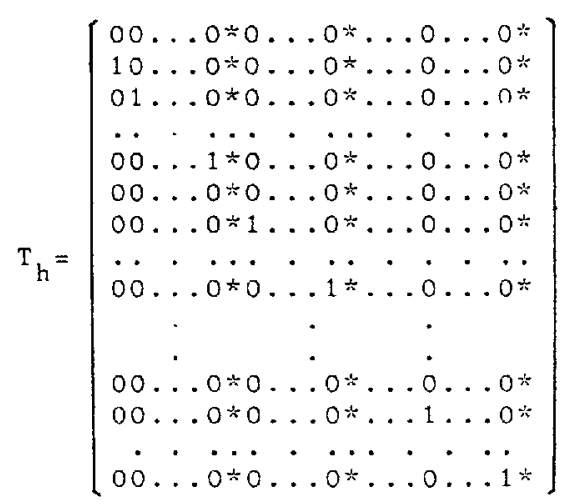

$$
T_{v}=\left[\begin{array}{cc}
00 \ldots 0 * & \ldots * \\
00 \ldots 0 * & \ldots * \\
\ldots & . \\
00 \ldots 0 * & \ldots * \\
10 \ldots 0 * & \ldots * \\
01 \ldots 0 * & \ldots * \\
. & . \\
. & . \\
00 \ldots 1 * & \ldots *
\end{array}\right]
$$

where the entries at $\div s$ ' positions are elements in $F_{2}$, then we say $A$ is an LR array of order $m \times n$. The window $A(0,0)$ (or $\mathbb{A}(0,0)$ ) is called tie initial state of $A$.

Definition 2.3: If an LR array of order mxn is also $a n$ m-array of order mxn, then we call it an LR m-array of order mxn.

Definition 2.4: Let $A=\left(a_{i j}\right)_{i \geqslant 0, j \geqslant 0}, \bar{B}=\left(b_{i j}\right)_{i \geqslant 0, j \geqslant 0}$ ze two periodic arrays. IE there exist two non-negative integers $p$, $q$ such that

$$
b_{i j}=a_{i+p, j+q} \quad \text { for } a 11 i \geqslant 0, j \geqslant 0
$$

then $B$ is called $(p, q)$-translation of $A$, denoted by $B=A, q$.

Obviously, the translation relation is an equivalence relation.

Proposition 2.1: Given $T_{h}, T_{v}$ as in $(2.2)$, let $G\left(T_{h}, T_{v}\right)$ be the set of all LR arrays with linear recurring relations $(2,1)$ and let $A, B \in G\left(T_{h}, I_{v}\right)$. Then

1) $A_{p, q} \in G\left(T_{h}, T_{v}\right) \quad$ for any integers $p, q \geqslant 0$.

2) Define $1 * A=A, 0 * A=0$. Then $G\left(T_{h}, T_{v}\right)$ is a vector space over $G F(2)$.

3) If there exists one LR m-array of order $m x n$ in $G\left(T_{h}, T_{v}\right)$, then every one in $G\left(T_{h}, T_{v}\right)$ is an LR m-array of order $m \times n$. Futhermore $T_{h} T_{v}=T_{v} T_{h}$ and $T_{h}, T_{v}$ are non-degenerate.

Definition 2.5: we call an array A non-degenerate, $: 5(2.1)$ holds for some nondegenerate matrices $T_{i_{1}}$ and $T_{v}$ as in (2.2).

Corollary 2.5.1: $\quad$ non-degenerate LR array must be geriodic.

Since we are interested in studying LR m-arrays, fran now on, we always assume that $T_{h}, T_{v}$ are non-degenerate and that they commute.

\section{3. $\alpha \beta-$ Array}

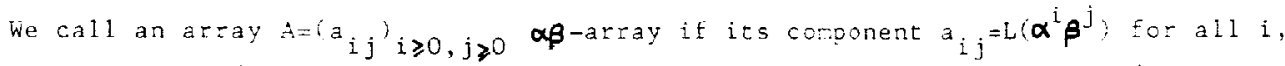
$j \geqslant 0$, where $\alpha, \beta \in G F(q)$, L is a linear function on $G F(q)$ over $G F(2)(G F(2) \subset G F(q))$.

In this section, we will mainly study linear recurzing relations of $\alpha \beta$-arrays and the necessary anc sufficient condition for an $\alpha \beta$-array to be an m-array. Ne will 
also compute the number of equivalence classes of $\alpha \beta$-m-arrays.

Lemma 3.1: Let $r s=2^{m n}-1,(r, s)=1, o(2 \bmod r)=m\left(i . e\right.$. the order of 2 in $\mathbb{Z}_{r}$ is $\left.m\right)$ or $\circ(2 \bmod s)=n$ and let $A=\left(a_{i j}\right)_{i \geqslant 0, j \geqslant 0}$, where $a_{i j}=L\left(\gamma^{i s+j 5}\right)$ for all $i \geqslant 0, j \geqslant 0$, L is a non-zero linear function on $G F\left(2^{m h}\right)$ over $G F(2), \gamma$ is a primitive element of $G F\left(2^{m n}\right)$. Then $A$ is an $(r, 5 ; m, n)$ LR m-array.

Proof: See [13].

Let $L$ be a non-zero function on the field $G F(q)$ over its prime field $G F(p)$. We define $L^{*}$ to be an elementwise transformation between vectors or matrices over $G F(q)$ and those over $G F(p)$ respectively as follows

$$
\left(a_{t}\right) L^{*}=\left(L\left(a_{t}\right)\right) \text { and }\left(a_{i j}\right) L^{*}=\left(L\left(a_{i j}\right)\right)
$$

where $\left(a_{t}\right)$ is a row or column vector over $G F(q)$ and $\left(a_{i j}\right)$ is a finite or infinite matix over $G F(q)$.

Proposition 2.2: Let $\alpha, \beta \in G F\left(2^{m}\right), o(\alpha)=r, o(\beta)=s$. If $r s=2^{m}-1$ for some $m$ and $(r, s)=1$, then there exists a primitive element $\gamma$ of $G F\left(2^{m}\right)$ such that $\alpha=\gamma^{S}$ and $\boldsymbol{\beta}=\boldsymbol{\gamma}^{r}$.

Theorem 3.3: Let $A=\left(\alpha^{i} \beta^{j}\right) L^{*}$ be an $\alpha \beta$-array, where $L$ is a non-zero linear function on $F_{2}(\boldsymbol{\alpha}, \boldsymbol{\beta})$. Then $A$ is a non-degenerate LR arrays. Furtermore, $A$ is an $(r, s ; m, n)$ m-array if and only if the following conditions are satisfied.

1) $o(\boldsymbol{\beta})=s, o(\boldsymbol{\alpha})=\mathrm{r}$ and $\mathrm{rs}=2^{\mathrm{mn}}-1$.

2) $\left\{\beta^{i} \alpha^{j} \mid 0<i<s, 0<j<r\right\}$ is the set of all non-zero elements of $G F\left(2^{m n}\right)$.

3) $\left\{\alpha^{i} \beta^{j}\{0 \leqslant i<m, 0 \leqslant j<n\}\right.$ is a basis of $G F\left(2^{m n}\right)$ over $G F(2)$.

In fact, $A$ is an $(r, s ; m, \pi)$ LR m-array.

Corollary 3.3.1: Let rxs be the period of an $\alpha \beta$-marray. Then $(r, s)=1$.

Let $f(x)=x^{m}+\sum_{i=1}^{m} c_{i} x^{m-i}$ be a monic polynomial of degree $m$ over $G F(2)$. Let $T=\left(d_{i j}\right)_{0 \leqslant i<m, 0 \leqslant j<n}$ be an mxn matrix over $G F(2)$ and $A=\left(a_{i j}\right)_{i \geqslant 0, j \geqslant 0}$ an arbitzary array. If

$$
\begin{aligned}
& a_{I+m, j}=\sum_{i=1}^{m} c_{i} a_{m+I-i, j} \\
& a_{I, J+n}=\sum_{i=0}^{a-1} \sum_{j=0}^{n-1} d_{i j} a_{I+i, J+j}
\end{aligned}
$$

we say $A \in G(f, I)$.

Proposition 3.4: Suppose $f, I$ as above. Then there exist $T_{h}, T_{v}$, such that $T_{h} T_{v}=T_{v} T_{h}, G\left(T_{h}, T_{v}\right)=G(E, T)$.

Proposition 3.5: Let $f, T$ be as in prop. 3.4. If all non-zero arrays in $3(E, T)$ are m-array of orde $=m \times n$, then $f(x)$ must be irreducible.

Proposition 3.6: Let $A \in G(f, T)$ be an m-array of order man and period $r \times 5$. Then $r=$ the period $p(E)$ of $E(x)$ and $o(2 \bmod r)=m$.

Proposition 3.7: If $r s=2^{m n}-1$, then either $o(2 \bmod r)=m n$ or $o(2 \bmod s)=m n$. 
Proposicion 3.8: Let $\mathrm{E}, \mathrm{T}$ be as in prop. 3.4, ail arrays in $\mathrm{G}(\mathrm{f}, \mathrm{T})$ be $(\mathrm{r}, \mathrm{s} ; \mathrm{m}, \mathrm{n})$ $m-a r r a y s, o(2 \bmod r)=m$ and $\alpha$ be a root of $f(x)$. Construct a polynomial $g(x)$ of degree n aver $F_{2}(\alpha)=G F\left(2^{m}\right)$ as follows:

$$
g(x)=x^{n}+\sum_{t=0}^{n-1} \sum_{t^{\prime}=0}^{m-1} d_{t^{\prime}, t^{\prime}} \alpha^{t^{\prime}} x^{t}
$$

then $g(x)$ is irreducible over $F_{2}(\alpha)$ and $p(g)=s$.

Theorem 3.9: Let $A=\left(L\left(\beta_{1}^{j} \alpha_{1}^{i}\right)\right)_{i \geqslant 0, j \geqslant 0}, B=\left(L\left(\beta_{2}^{j} \alpha_{2}^{i}\right)\right)_{i \geqslant 0, j \geqslant 0}$ be two $\alpha \beta-m-a r r a y s$ of period rxs. Then $A$ and $B$ are equivalent if and only if the following statements are satisfied.

1) $\alpha_{1}$ and $\alpha_{2}$ are conjugate over GF(2).

2) if $\alpha_{1}=\alpha_{2}^{2^{i_{0}}}$ (for some $i_{0}$ ), then $\beta_{1}$ and $\beta_{2}^{2^{i_{\infty}}}$ are conjugate over $F_{2}\left(\alpha_{1}\right)=F_{2}\left(\alpha_{2}\right)$

Theorem 3.10: The number of equivalence classes of $\alpha \beta-m-a r r a y s$ of period rxs is $\phi(r s) / \log _{2}(r s+1)$, where $\phi$ is Euler function.

4. General LR m-Array

In this section, we discuss general LR m-arrays. The main results are about their structure, enumeration and the necessary and sufficient conditions for existence of arrays with given period rxs.

Proposition 4.1: Suppose $A \in G\left(T_{h}, T_{v}\right)$ is an $(r, 5 ; n, n)$ LR m-array. Then $p\left(T_{h}\right)=s$, $p\left(T_{v}\right)=r$ and the order of any eigenvalue of $T_{h}\left(T_{v}\right.$ res?.) is $s(r$ resp.).

Proposition 4.2: Suppose $A \in G\left(T_{h}, T_{v}\right)$ is an $(r, s ; m, n)$ LR m-array and $o(2 \bmod s)=m n$. Then

1) the characteristic polynomial of $T_{h}$ is irteducible, and both $T_{h}$ and $T_{v}$ are similar to a diagonal form under same transzormation.

2) the minimal polynomial $g(x)$ of $T_{v}$ is irrecucible and $\operatorname{deg}(g(x))=m^{\prime} i f$ or $(2 \mathrm{mod}$ $r)=m$.

Theorem 4.3(Existence): For given positive integers $r$ and $s$, there exists an m-array with period rxs, if and only if $(r, s)=1$ and $s s=2^{m}-1$ (for some $m$ ).

Theorem 4.4(Stzucture): Any LR m-array must be $\exists n \alpha \beta-n-a r r a y$.

Remark 4.5: By Prop. 3.2, we know that there is a primitive element $\gamma$ in $G F\left(2^{m n}\right)$ such that

$$
A=\left(L\left(\gamma^{i s-j r}\right)\right) ; \geqslant 0, j \geqslant 0
$$

Therefore each LR ä-aray can be determined by a prinitive element $\gamma$ and a 1 inear function $L$. We denote $t$ by $A_{r \times s}(\gamma, L)$, where $r x s$ is the period of A. Obviously, for different linear functions, $A_{r \times s}(\gamma, L)$ 's are equivaient.

Corollary 4.4.1: An $(r, s ; \pi, \pi)$ LR m-array is aiso an $(r, s ; m n, 1)$ or $(r, s ; 1, m n) L R$ 
m-array according which one of $o(2 \bmod r)$ and $o(2 \bmod s)$ is $\mathrm{mn}$.

Corollary 4.4.2: The number of equivalence classes of LR m-arrays of period rxs is $\phi(r s) / \log _{2}(r s+1)$.

Remark 4.6: By Prop. 3.9, it is easy to prove that, for any two conjugate primitive elements $\gamma_{1}$ and $\gamma_{2}$ of $G F\left(2^{\mathrm{mn}}\right)$ with respect to $G F(2), A_{I \times s}\left(\gamma_{1}, L\right)$ and $A_{\text {IXs }}\left(\gamma_{2}, L\right)$ are equivalent. But the number of conjugate classes of primitive elements of $G F\left(2^{\mathrm{mn}}\right.$ ) with respect to $\mathrm{GF}(2)$ is also $\phi(r s) / \log _{2}(r s+1)$, so that there is a 1 - 1 correspondence between the equivalence classes of $r \times s$ periodic LR m-arrays and the conjugate classes of primitive elements of GF $\left(2^{\mathrm{mn}}\right.$ ) (or all primitive polynomials of degree mn over GF( 2)) (see Remark 4.5 and Corollary 4.4.2). This map can be obtained by (4.1) of Remark 4.5.

The above correspondence is very powerful in Section 5 for studying the properties of LR m-arrays. From now on, $G_{\text {rxs }}(f)$ will denote the set of all the arrays of period rxs which are corresponded to a primitive polynomial f.

5. Properties of LR m-Arrays

LR m-arrays can be thought of as generalized m-sequences. LR m-arrays have many good properties, as m-sequences do. In this section, we study the properties of translation-addition, sampling and correlation.

Proposition 5.1: An infinite matrix A of period rxs is an LR m-array if and only if

1) $(r, s)=1$

2) For any given integers $p_{1}, p_{2}, q_{1}, q_{2} \geqslant 0$, either $A_{p_{1}}, q_{1}+A_{p_{2}}, q_{2}=0$ or $=A_{p}, q$ for

The property given above is a characteristic property of LR m-arrays called the translation-addition property of LR m-arrays.

Proposition 5.2: For any LR m-array of order mxn, the mn vectors $\bar{A}(i, j)(0 \leqslant i<m$, $0 \leqslant j<n)$ are linearly independent and all $\mathbb{A}(i, j)$ can be linearly expressed by them.

Definition 5.1: Let $A=\left(a_{i j}\right)_{i \geqslant 0, j \geqslant 0},(r, s)$ be a pair of positive integers. We $\operatorname{call} A^{(r, s)}=\left(a_{i \tau, j s}{ }_{i \geqslant 0, j \geqslant 0}\right.$ an $(r, s)-$ sample of A. Especilly, $A^{(t, t)}$ is called a diagonal sample of A.

Theorem 5.3: Let A be an LR array with period $\mathrm{P}_{\mathrm{V}} \times \mathrm{P}_{\mathrm{h}}$ and $(\mathrm{r}, \mathrm{s})$ be a pair of posicive integers. If $\left(=, P_{v}\right)=1=\left(s, P_{h}\right)$, then $A(r, s)$ is again an LR m-array with period $P_{V} \times P_{h}$ and any $L R$ m-array of period $P_{v} \times P_{h}$ are equivalent to some (diagonal) sample of A. Furthermore, if $\left(r^{\prime}, P_{v}\right)=\left(r, P_{v}\right)=\left(s^{\prime}, P_{h}\right)=\left(s, p_{h}\right)=1$, then $A^{(r, s)}$ and $A^{\left(r^{\prime}, s^{\prime}\right)}$ are equivalent if and only if

$$
r^{\prime} \equiv \mathrm{r} 2^{t} \bmod 2^{m}-1 \text { and } s^{\prime} \equiv s 2^{t+m n t^{\prime}} \bmod 2^{m n}-1 \text { for some } t \text { and } t^{\prime}
$$

Definition 5.2: Let $A=\left(a_{i j}\right)_{i \geqslant 0, j \geqslant 0}$ be an array of period rxs. The autocorrelation 
function of $A$ is defined as the function



where $\eta$ is a function from $G F(2)$ to $\{1,-1\}$ such that $\eta(0)=1, \eta(1)=-1$.

Difinition 5.3: Let $A$ be a binary array with period $r$ s. If

$$
c_{A}(p, q)= \begin{cases}r s & \text { when } p \equiv 0 \bmod x \text { and } q \equiv 0 \bmod s \\ -1 & \text { others }\end{cases}
$$

then we call A a pseudo-random array.

Theorem 5.4: Suppose A is a pseudo-random array with period rxs. Then rs 3 mod 4 and the difference between the numbers of $1^{\prime} s$ and $O^{\prime} s$ in a period of $A$ is 1 .

Theorem 5.5: Any LR m-array is a pseudo-random array.

Definition 5.4: Let $A=\left(a_{i j}\right)_{i \geqslant 0, j \geqslant 0}, B=\left(b_{i j}\right)_{i \geqslant 0, j \geqslant 0}$ be two arrays of period rxs. Define their crosscorrelation function as follows:

$$
\left.C_{A, B}: 2 \times Z \rightarrow Z: \quad C_{A, B}(p, q)=\sum_{i=0}^{r-1} \sum_{j=0}^{s-1} \quad \eta_{i j}^{\left(a_{i j}\right)} \quad \eta_{i+p, j+q}^{(b}\right)
$$

where $\eta$ is just as in Definition 5.2 .

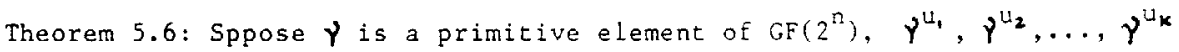
$\left(0<k<2^{n}-1\right.$ ) are the Eirst roots of primitive polynomials $f_{u_{1}}(x), \ldots, f_{u_{k}}$ ( $x$ ) respectively, $u_{1}>u_{2}>\cdots>u_{k},(r, s)=1$, Is $=2^{n}-1$. Then for any arrays $A \in G_{r \times s}\left(E_{u_{i}}\right), B \in G_{r \times s}$ ( $\left.f_{u}\right)$ and any $t_{1}, t_{2} \geqslant 0$, we have

$$
C_{A, B}\left(t_{1}, t_{2}\right) \leqslant 2^{n}-1-2 u_{k}
$$

Theorem 5.7(gold Optimum Pair): Let $\gamma$ be a primitive element of $G F\left(2^{n}\right)$.

$$
\begin{aligned}
& u_{1}=2^{n-1}-1
\end{aligned}
$$

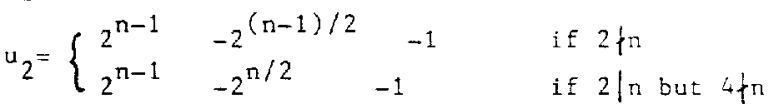

and $(r, s)=1, r s=2^{n}-1$. Then for any $A \in G_{r \times s}\left(f_{u_{1}}\right), B \in G_{r \times s}\left(f_{u_{2}}\right)$ and $t_{1}$, $t_{2} \geqslant 0$, we have:

$$
C_{A, B}\left(t_{1}, t_{2}\right)= \begin{cases}2(n+1) / 2+1 & \text { if } 2 \nmid n \\ 2(n+2) / 2+1 & \text { if } 2 / n \text { but } 4 \nmid n\end{cases}
$$

\section{REFERENCE}

[1]. Zhe-xian Wan, "Algebra and Coding Theory." Science Press, Beijing. 1980, revised edition.

[2]. B. Gordon, "On the existence of perfect maps" IEEE Trans. Inform. Theory Vol. IT-12 486-487 1966.

[3]. F.J. Macwilliams and N.J.A. Sloane, "Pseudo-randon sequences and arrays". Proc. 
IEEE vol.64 pp 1715-1729. 1976.

[4]. T. Normura, H. Miyakawa, H. Imai and A. Fukuda, "A theory of two dimensional linear recurring arrays". IEEE Trans. Inform. Theory vol. IT-18 pp 775-785, 1972 .

[5]. R. Spann, "A two-dimensional correlation property of pseudo-random maximallength sequences". Proc. IEEE vol.53 pp $2137,1963$.

[6]. J. H. van Line, F. J. Macwilliams and N. J. A. Sloane, "On pseudo-random arrays". SIAM J. Appl. Math. vol 36 pp 62-72, 1979.

[7]. C. T. Fan, S. M. Fan, S. L. Ma and M. K. Siu, "On de-Bruijn arrays". ARS Combin. vol. 19A (1985), 205-213.

[8]. S. Homer, Jerry Goldman, "Doubly-periodic sequences and two-dimensional recurrence", SIAM J. Alg. disc. Math. vol 6 (1985), 360-370.

[9]. S.L. Ma, "A note on binary arrays with a certain window property", IEEE Tran. Inform. Theory vol IT-30 (1984), 774-775.

[10]. T. Nomura and A. Fukuda, "Linear recurring planes and two-dimensional cyclic codes" Trans. Inst. Electron. Commun. Eng. Jap. vol. 54-A pp 147-154 Mar. 1971

[11]. I. S. Reed and R. M. Stewart, "Notes on the existence of perfect maps: IEEE Trans. Inform. Theory vol. IT-8 pp 10-12 Jan. 1962.

[12]. D. Calabro and J. K. Wolf, "On the synthesis of two-dimensional arrays with desirable correlation properties:, Inform. Contr. vol. 11 pp 537-560 Nov/Dec. 1967 .

[13]. M. K. Siu, "m-Arrays and M-Arrays." (1985).

[14]. L. E. Diccson, "On the cyclatomic function', Amer. Math. Monthly vol. 12 (1905) $86-89$. 